\section{On the Dependence of Magnetic Stochastic Resonance Features on the Features of Magnetic Hysteresis}

Mantegna, Rosario N. ${ }^{\text {ISpagnolo, Bernardo }}$; Testa, Luigi ${ }^{2}$; Trapanese, Marco ${ }^{2}$ 1 INFM, Unità di Palermo and Dipartimento di Fisica e Tecnologie Relative Universita di Palermo, Palermo, Italy.

2 Dipartimento di Ingegneria Elettrica, Università di Palermo, Palermo, Italy

Introduction

Stochastic resonance (SR) is generally considered as an enhancement of the system response for certain finite values of the noise strength $[1,2]$. In particular the signal to noise ratio (SNR) and the signal amplification show a maximum as a function of the noise intensity.

This effect has been experimentally observed in many physical systems and also in magnetic systems [3,4]. However, as far as magnetic systems are concerned, a clearer relation between the features of the hysteresis and the features of SR is needed in order to be able to foresee the behaviour of a magnetic material in which SR might occur.

In order to clarify this relation, in this paper we numerically study Magnetic Stochastic Resonance in several magnetic systems having different hysteresis loop. We model the various hysteresis loops by using the scalar Preisach model in which several identification functions are used. The use of Preisach model allows us to study the features of the SR in comnection with the parameters used in Preisach model. More particularly, we show that:

- there are magnetic systems in which there is a resonant behaviour both in the signal amplification and in SNR;

- there are magnetic systems in which there is a resonant behaviour in the signal amplification but no resonance is present in SNR:

- there are magnetic systems in which SR doesn't occur in a meaningful way.

The Preisach Mode!

The model that has been used in this paper to simulate the hysteresis loop is the scalar Preisach model [5]. The global magnetization of the magnetic material can be obtained from the superposition of the magnetization of many particles (called hysterons). The characteristics of a Preisach particle can be described on a plane, called Preisach plane. A generic magnetic material can be described by locating the hysterons on suitable parts of the plane. Under the assumption that the number of particles in an area of Preisach plane is described by a Preisach density function $p\left(h_{m}, h_{c}\right)$, the global magnetization of the magnetic material is:

$M=2 M_{c} \int_{0}^{\infty} d h_{m} \int_{0}^{b\left(\lambda_{m}\right)} p\left(h_{m}, h_{c}\right) d h_{c}$

where $M$ is the global magnetization, $b\left(h_{m}\right)$ is the limit that is spanned by the density function and $h_{c}$ and $h_{m}$ are two fields that describe the behaviour of a single hysteron.

A suitable form for the Preisach function is the Lorentzian function.

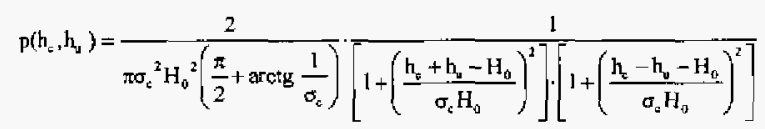

In this work we study the features of SR in many magnetic systems by varying the parameters in the Preisach distribution.

\section{The equation}

In our approach, we assumed that the extemal magnetic field ( $\left.h_{\mathrm{ex}}\right)$ applied to a magnetic material consisted of two components: one small sinuisodal component added to a gaussian noise component:

$h_{\mathrm{ext}}=H_{\mathrm{t}} \sin l+D(t)$

where $t$ is the time and $D$ is the gaussian noise. The value of $h$ was computed at several time steps. As a result the history of the magnetization of our system could be computed by using eq (3) in the Preisach Model. The evolution of the magnetization can be evaluated at each time step by eq. 1 ; by doing so we neglect any relaxation effect

The numerical results.

We computed the SNR, the power amplification and the behaviour of the magnetization for several $\mathrm{H}_{5}$ and $\mathrm{D}$ and with various parameters in the Preisach function (i.e. with different hysteresis loops). In these digest, we show how stochastic resonance depends on the parameter $\sigma \mathrm{c}$ and on $\mathrm{H}_{0}$.

From fig. 1 it can be seen that in a system in which se has a relatively small value, $S R$ is present both in SNR as well in the signal amplification. At high values of sc the stochastic resonance is present only in the signal amplification (fig.2).

From fig. 3 it can be seen that the effect of $\mathrm{H}_{0}$ is to shift the maximum of $\mathrm{SR}$.

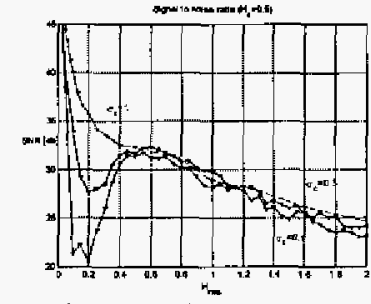

Fig. I SNR. It can be seen how SR occurs up to sc $<1\left(H_{0}=1\right)$

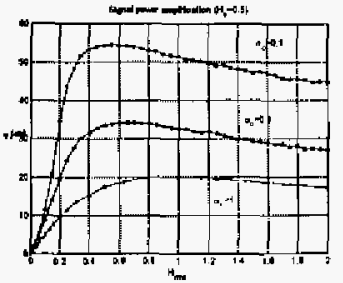

Fig.2 Signal amplification. It can be seen how SR occurs for all se chosen . $\left(\mathrm{H}_{0}=1\right)$

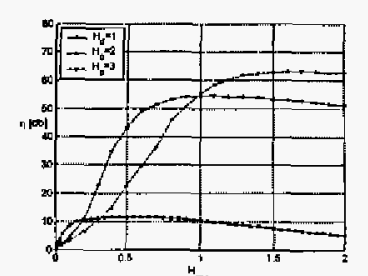

Fig. 3 SNR for different values of $H$ at fixed $\sigma_{\mathrm{c}}(=0.5)$

\section{Conclusions}

In this paper we showed as SR depends on the parameters of Prejsach function. We showed how SR is affected from the parameters of the Preisach function chosen. More particularly, our numerical approach show how the field $\mathrm{H}_{0}$ shifts the on set of SR and how a large dispersion of the distribution of hysterons degrades the SR.

\section{References}

[1] R. Benzi, A. Sutera, and A. Vulpiani, J. Phys, A 14, 453 (1981)

[2] R. Benzi, G. Parisi, A. Sutera and A. Vulpiani, Tellus 34, 10, (1982).

[3] Grigorenko and P.I, Nikitin, JEEE Trans. on Magnetics, 31, 2491 (1995)

[4] R.N.Mantegna, B.Spagnolo, L.Testa, M.Trapanese to be published.

[5] F.Preisach, Zeitschrift furr Physik, B 94, 277 (1935). 